\title{
Design and Evaluation of the Constant-Flux Inductor with Enclosed-Winding
}

\author{
Han Cui and Khai D. T. Ngo \\ The Bradley Department of Electrical and Computer \\ Engineering \\ Virginia Polytechnic Institute and State University \\ Blacksburg, VA 24061, USA
}

\author{
Jim Moss, Michele Lim, and Ernesto Rey \\ Texas Instruments Inc. \\ Santa Clara, CA 95051 USA
}

\begin{abstract}
The "constant-flux" concept introduced recently is leveraged to distribute magnetic flux to improve energy density, lowering the profile of an inductor. The optimal flux distribution with normalized parameters is identified mathematically, and verified by simulation. It is then applied to reduce the de resistance of a commercial inductor by a factor of two, keeping the outer dimensions and inductance the same. Thermal-limited current rating is improved by $\mathbf{5 0 \%}$, whereas saturation-limited current rating is improved by $20 \%$ thanks to the suppression of flux crowding. The simulation result is verified by measurement results of a prototype under high current bias.
\end{abstract}

\section{INTRODUCTION}

In order to improve energy density, a variety of techniques have been reported in the literature [1-9] to minimize inductor volume without sacrificing performance. The matrix inductor from [2-4] has low profile and good heat dissipation, but it encounters non-uniform flux density among elements which results in poor space utilization. With the purpose of fully utilizing magnetic material and achieving low profile inductor, the multi-permeability structure was discussed in [5] that employs changing permeability to achieve uniform flux density; coupled inductors with lateral flux structure discussed in [6] were proved to have higher density than vertical flux structure. Recently, the concept of constant-flux inductor (CFI) was demonstrated in [7] aiming at improving the space utilization by making the flux uniform throughout the core volume. Based on the position of the core and the winding, the design of constant-flux inductor with enclosed-core geometry, such as toroids, was introduced in [8]; inductor with enclosed-winding geometry, such as injection-molded inductor, was discussed in detail in [9]. By following the design procedures given by [9], the flux distribution inside the core material is regulated by the uniformity factor $\alpha$. As shown in Figure 1, by eliminating the high-flux regions in the core of a commercial inductor, the total volume of the commercial inductor can be reduced by a factor of two while keeping the inductance and resistance the same.

This work was supported by Texas Instruments and National Science Foundation under Grant No. ECCS-1231965.
Based on the model for the constant-flux inductor constructed in [9], this paper focuses on the design procedure of constant-flux inductors with normalized parameters in order to achieve the optimal performance and studies the impact of uniform flux distribution on performance metrics with comparisons to commercial inductors.

Section II demonstrates the procedure to normalize all the design parameters and shows the impact of the uniformity factor and inductor size on the design. Section III evaluates the performance metrics such as energy, dc winding loss, dc quality factor. A detailed design procedure using the normalized parameters is given in Section IV to generalize and simplify the design. Section $\mathrm{V}$ compares the current ratings of a commercial counterpart within the same volume and inductance as a constant-flux inductor and the simulation result is verified by experiments in Section VI. Conclusions are given in Section VII.

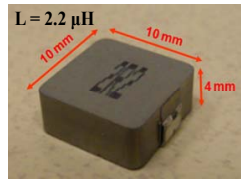

(a)

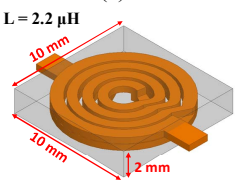

(c)

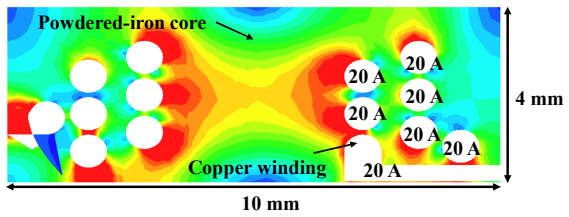

(b)

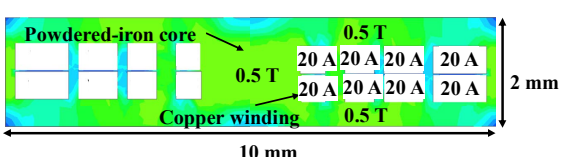

(d)
Figure 1. (a) A commercial enclosed-winding inductor with inductance of $2.2 \mu \mathrm{H}$, resistance of $7 \mathrm{~m} \Omega$, height of $4 \mathrm{~mm}$, and permeability of $35 \mu_{0}$; (b) cross-sectional view of the commercial inductor showing unevenly distributed flux density inside the core; (c) 3D structure of constant-flux inductor discussed in [9] with winding enclosed by core; total thickness is reduced by half to $2 \mathrm{~mm}$, whereas inductance and resistance are kept at $2.24 \mu \mathrm{H}$ and $5.5 \mathrm{~m} \Omega$ with $35 \mu_{0}$ permeability; (d) relatively uniform distribution of flux density in the core of the constant-flux inductor. 


\section{NORMALIZED DESIGN WITH LINEAR MAGNETIC CORE}

The behavioral model of constant-flux inductor was constructed in [9]. With given specifications, winding configuration with uniform flux distribution can be achieved by following the design equations and procedures. In order to generalize the design procedure and to analyze the impacts of design parameters systematically, the model with normalized parameters is discussed in this section.

\section{A. Bases for Normalization}

The parameters such as dimensions, Ampere-turns, energy, winding loss, inductance, and resistance shown in TABLE I are normalized to a base function such that the whole model of constant-flux can be analyzed and evaluated regardless of the inductor volume, material's property, current rating, etc.

TABLE I. BASES FOR NORMALIZATION

\begin{tabular}{|c|c|c|}
\hline Symbol & Used for & Mathematical Expression \\
\hline$R_{\text {base }}$ & $R_{O j}, R_{I j}$ in (7) & $R_{\text {base }}=R_{c}$ \\
\hline$A T_{\text {base }}$ & $A T$ in (14) & $A T_{\text {base }}=B_{\max } \cdot 2 \pi \cdot R_{c} / \mu$ \\
\hline$E_{\text {base }}$ & $E$ in (16) & $E_{\text {base }}={B_{\max }}^{2} \cdot \pi \cdot R_{c}^{3} / 2 \mu$ \\
\hline $\begin{array}{l}\text { loss_base } \\
\end{array}$ & $P_{\text {loss }}$ in $(20)$ & $P_{\text {loss_base }}=A T_{\text {base }}^{2} \cdot R_{N_{-} \text {base }}=4 \pi B_{\max }^{2} \rho R_{c} / \mu^{2}(4)$ \\
\hline$A_{L_{-} \text {base }}$ & $L$ in $(23)$ & $A_{L_{-} \text {base }}=2 E_{\text {base }} / A T_{\text {base }}^{2}=\mu \cdot R_{c} / 4 \pi(5)$ \\
\hline$R_{N_{-} \text {base }}$ & $R_{d c}$ in $(23)$ & $R_{N_{\text {base }}}=P_{\text {loss base }} / A T_{\text {base }}^{2}=\rho / \pi R_{c}$ \\
\hline
\end{tabular}

\section{B. Geometric Variables to Program Flux Distribution}

Figure 2 shows the axisymmetric view of a constant-flux inductor with three winding windows. The objective of constant-flux design is to synthesize the Ampere-turns and radii of each winding window to constrain the flux variation to a "uniformity factor $\alpha$ ", ratio of the minimum flux density $\alpha B_{\max }$ to the maximum flux density $B_{\max }$ allowed around winding window $j$. According to the behavioral model presented in [9], the field equations (1) - (4) can be constructed based on Ampere's law and Gauss' law to solve for the geometrical variables such as the radii and thickness of the winding windows. To ensure the inner radius of the innermost winding window satisfy the equations, a center hole should be included to obey the field distribution. The innermost radius of $N_{w}$ winding window designs (such as $R_{O 4}$ in Figure 2) is determined by

$$
R_{O(j+1)}^{2}+2 R_{I j} H_{p}-R_{I j}^{2}=0, \quad j=N_{w}
$$

where $R_{O j}$ and $R_{I j}$ are the outer and inner radius of winding window $j$, respectively; $H_{p}$ is the plate thickness shown in Figure $2 ; N_{w}$ is the number of winding windows.

To exclude the impact of the dimensions on inductor performance, the geometrical parameters such as the radii $R_{O j}, R_{I j}$, and the plate thickness $H_{p}$ are normalized to the outer radius of the core $R_{c}$ as follows:

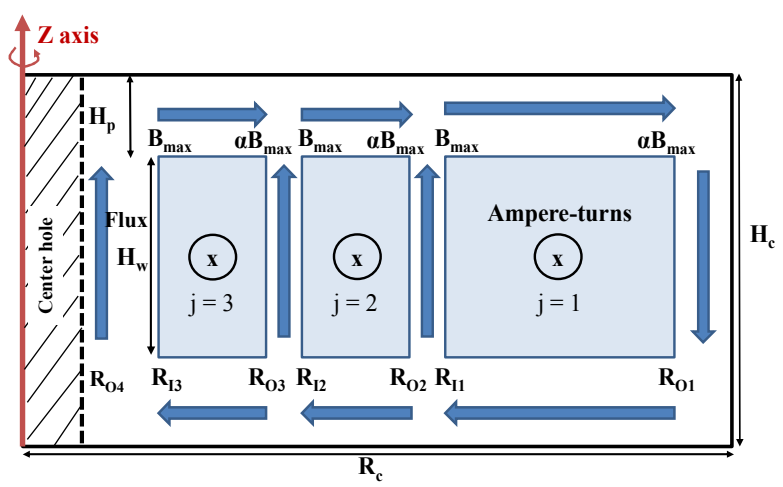

Figure 2. Axisymmetric view of a constant-flux inductor with enclosed winding showing three winding windows, the center hole, Ampere-turns direction, and magnetic flux path.

$$
\begin{aligned}
& h_{c}=H_{c} / R_{\text {base }}, h_{p}=H_{p} / R_{\text {base }}, r_{l j}=R_{l j} / R_{\text {base }}, \\
& r_{O j}=R_{O j} / R_{\text {base }}, h_{w}=\left(H_{c}-2 H_{p}\right) / R_{\text {base }}=h_{c}-2 h_{p}
\end{aligned}
$$

Based on the normalized geometrical parameters, the field equations (1) - (4) in [9] and (7) can be rewritten and solved in the normalized form regardless of the real dimensions of the core:

$$
\begin{gathered}
1-r_{O 1}^{2}-2 r_{O 1} h_{p}=0 \\
\left(h_{c}-2 h_{p}\right) \cdot(1+\alpha)-2 \cdot r_{I 1} \cdot \ln (\alpha)-\alpha \cdot h_{c} \cdot(1+\alpha) \\
+2 \cdot \alpha \cdot r_{O 2} \cdot \ln \left(r_{O 2}\right)=0 \\
r_{I j}=\alpha r_{O j}, \quad 1 \leq j \leq N_{w} \\
r_{O(j+1)}^{2}-2 \alpha r_{O(j+1)} h_{p}+2 r_{I j} h_{p}-r_{I j}^{2}=0, \quad 1 \leq j \leq N_{w}-1 \\
r_{O(j+1)}^{2}+2 r_{I j} h_{p}-r_{I j}^{2}=0, j=N_{w}
\end{gathered}
$$

Equations (9) - (12) simultaneously solve the four variables $\mathrm{r}_{o l}, r_{i l}, r_{o 2}$, and $h_{p}$ for the first winding window $j=$ 1 and recursively yield the radii of the remaining winding windows $j>1$ together with (13).

Figure 3 shows the solutions of (9) - (13) when uniformity factor $\alpha$ is changed from 0.5 to 0.8 , parametric with $h_{c}$ ranged from 0.2 to 1 . For $h_{c}$ smaller than 0.2 or larger than 1, it is impractical to have constant-flux designs. For designs with $N_{w}\left(N_{w}=1,2,3,4\right)$ winding windows, the normalized radii of the winding windows can be read from Figure 3(a) and Figure 3(b) directly; the innermost radius $R_{O j}\left(j=N_{w}+1\right)$ of the center hole can be read from Figure 3(c), and the plate thickness regardless of $N_{w}$ can be obtained from Figure 3(d). As shown in Figure 3(a) and Figure 3(b), larger $\alpha$ always leads to thinner magnetic walls between adjacent winding windows, narrower window width, and smaller plate thickness. Thus, $\alpha$ is usually selected considering fabrication clearances. It is possible that for a certain $h_{c}$ and $\alpha$, no solution exists for (9) - (13); therefore, Figure 3 contains curves that do not span the entire range of $\alpha$. 


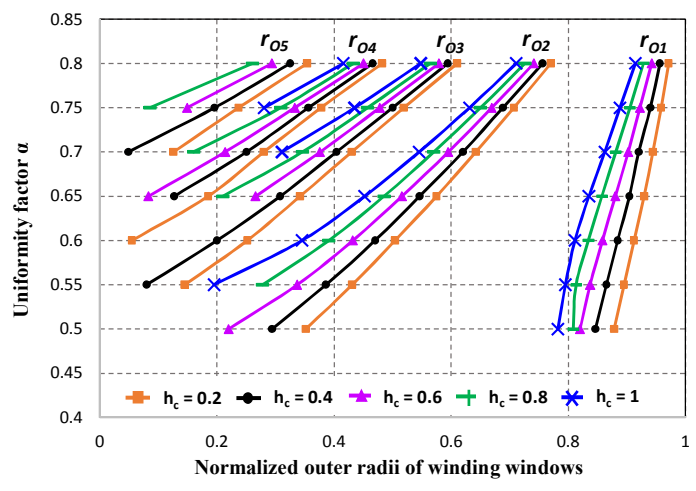

(a)

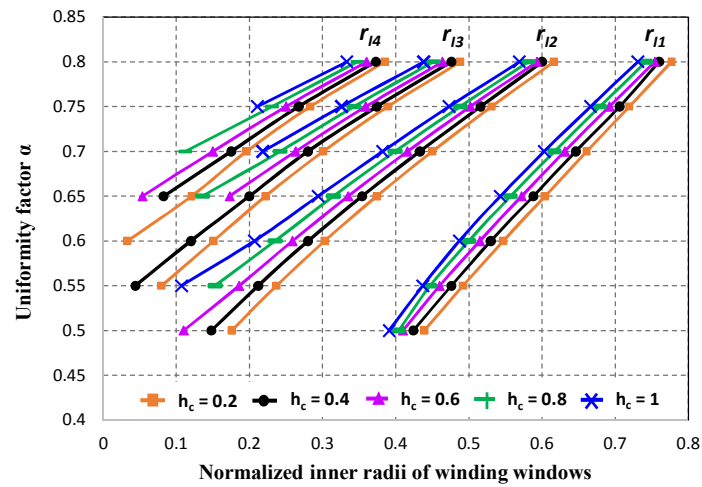

(b)

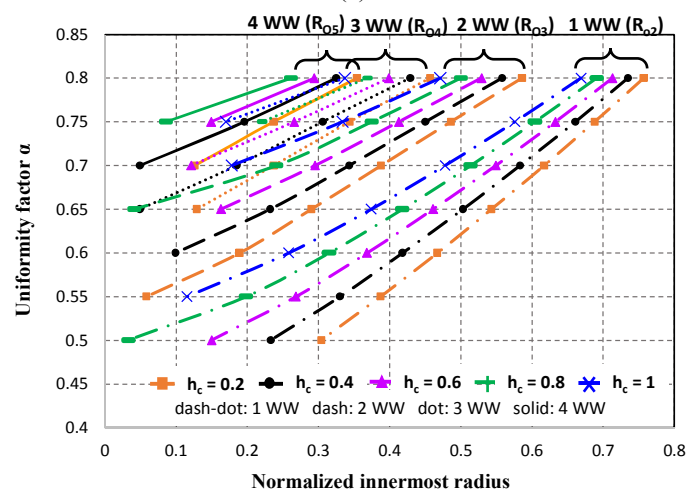

(c)

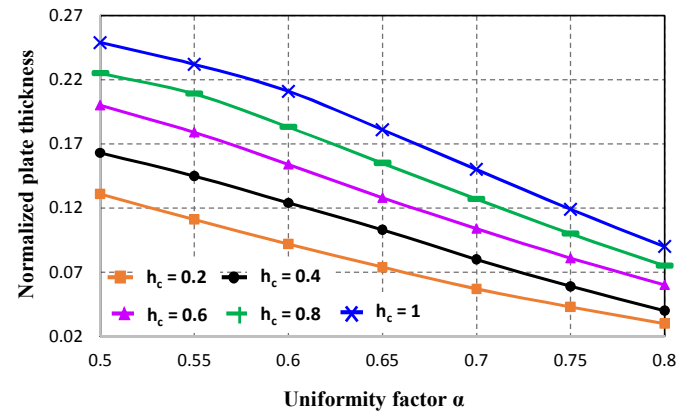

(d)

Figure 3. Uniformity factor versus the (a) outer radii, (b) inner radii, (c) innermost radius (center hole radius), and (d) plate thickness calculated from (9)-(13) for one to four winding windows, parametric with $h_{c}$ from 0.2 to 1 .

\section{Distributed and Equivalent Ampere-Turns}

The structures of the winding windows are fixed after the radii and plate thickness are determined. As presented in [9], the total Ampere-turns is the sum of $A T_{j}$ for each winding window which is determined by Ampere's law:

$$
\begin{aligned}
& A T=\sum_{j=1}^{N_{w}} A T_{j} \\
& =\mu^{-1} \cdot \sum_{j=1}^{N_{w}}\left[H_{w} \cdot\left(B_{\max } \pm \alpha B_{\max }\right)-2 \cdot B_{\max } \cdot R_{I j} \cdot \ln (\alpha)\right]
\end{aligned}
$$

The normalized Ampere-turns is given by

$$
A T_{\text {norm }}=\frac{A T}{A T_{\text {base }}}=\frac{1}{2 \pi} \cdot \sum_{j=1}^{N_{w}}\left[h_{w} \cdot(1 \pm \alpha)-2 \cdot r_{I j} \cdot \ln (\alpha)\right]
$$

The normalized Ampere-turns under different $\alpha, h_{c}$, and number of winding windows are demonstrated in Figure 4. Each curve shows the total normalized Ampere-turns in $N_{w}$ $\left(N_{w}=1,2,3,4\right)$ winding windows. For each $h_{c}$, the difference between the curves for $N_{w}$ and $\left(N_{w}-1\right)$ winding windows is the Ampere-turns in window $N_{w}{ }^{t h}$. Either larger number of winding windows or larger core volume requires more Ampere-turns to maintain the uniformity level, which means more turns are needed under the same current rating.

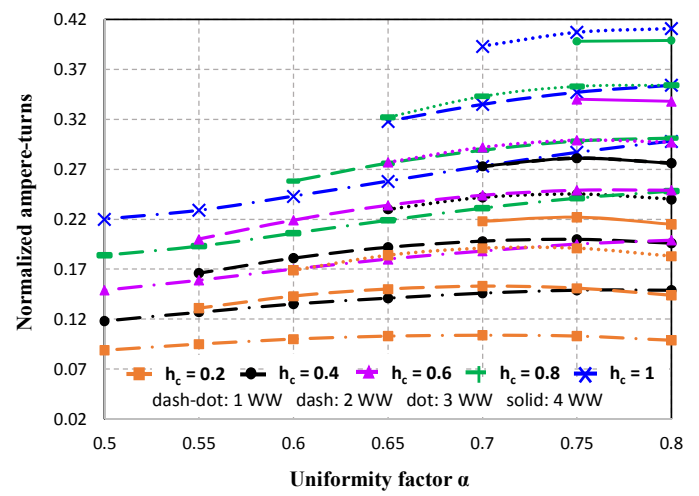

Figure 4. Impact of uniformity factor $\alpha$ on normalized Ampere-turns calculated from (15) using normalized dimensions shown in Figure 3 for one to four winding windows, parametric with $h_{c}$ from 0.2 to 1 .

\section{PERFORMANCE EVALUATION}

\section{A. Magnetic Energy and Inductance-per-Turn ${ }^{2}$}

The energy can be calculated based on the ideal assumption that the flux density is uniformly distributed throughout the core volume at an average value that is related to the uniformity factor $\alpha$. Therefore, the energy stored in the core is determined by integrating the average flux density:

$$
E \approx(2 \mu)^{-1} B_{\text {avg }}{ }^{2} \pi\left[\left(R_{c}^{2}-R^{2}{ }_{O\left(N_{w}+1\right)}\right) H_{c}-H_{w} \sum_{j=1}^{N_{w}}\left(R_{O j}^{2}-R_{l j}^{2}\right)\right]
$$

With the purpose of deriving an accurate equation for the average flux density $B_{a v g}$ versus $\alpha$, designs with various $h_{c}$ and $\alpha$ were simulated using the geometrical parameters from Figure 3 and current excitations from Figure 4. For each simulation, the average flux density was calculated 
from the total energy simulated, and the simulated result of the average flux density after normalized to $B_{\max }$ is shown in Figure 5. Under the same uniformity factor, although the design has variable number of winding windows and $h_{c}$, the average flux density almost remains the same within a range of $5 \%$ difference since the field is strictly regulated by the equations.

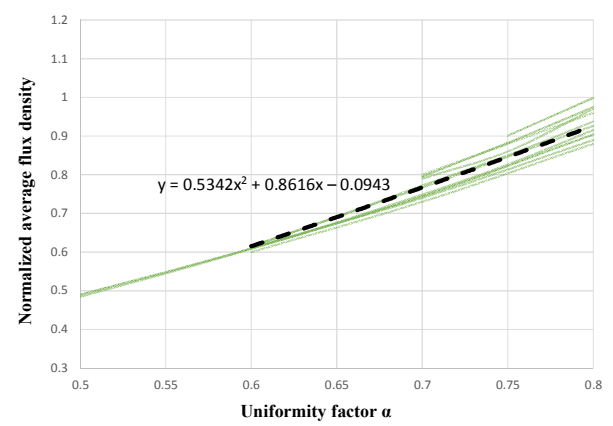

Figure 5. Normalized average flux density versus uniformity factor from simulations for one to four winding windows, parametric with $h_{c}$ from 0.2 to 1 .

Therefore, the relationship between average flux density and uniformity factor is curve-fit as

$$
B_{\text {avg_norm }}=B_{\text {avg }} / B_{\max }=0.5342 \alpha^{2}+0.8616 \alpha-0.0943
$$

Then the normalized energy takes the form

$$
\begin{aligned}
& E_{\text {norm }}=E / E_{\text {base }}=\left(0.5342 \alpha^{2}+0.8616 \alpha-0.0943\right)^{2} . \\
& \left(h_{c}\left(1-r_{O\left(N_{w}+1\right)}^{2}\right)-h_{w} \sum_{j=1}^{N_{w}} r_{O j}^{2}-r_{I j}^{2}\right)
\end{aligned}
$$

The benefit of the normalized energy is that it is independent of the material's property and only relates to the inputs $h_{c}$ and $\alpha$. From (18), when the normalized core thickness $h_{c}$ varies from 0.2 to 1 , and the number of winding windows changes from $1-4$, the relationship between the normalized energy and the uniformity factor $\alpha$ is shown in Figure 6(a). Every curve of the normalized energy has a parabolic shape caused by the change in $\alpha$ and corresponding winding geometry. When $\alpha$ is close to 0.5 , the uniformity level is very low, limiting the energy that can be stored in the core; on the other hand, plate thickness is thinner as shown in Figure 3(d) when $\alpha$ is close to 0.8, leaving smaller core volume to energy storage and reducing the energy density.

The inductance-per-turn ${ }^{2}$ is related to the normalized energy and Ampere-turns by

$$
\begin{aligned}
& A_{L_{-} \text {norm }}=A_{L} / A_{L_{-} \text {base }}=E_{\text {norm }} / A T_{\text {norm }}^{2} \\
= & \frac{\left(0.5342 \alpha^{2}+0.8616 \alpha-0.0943\right)^{2}\left[h_{c}\left(1-r_{O\left(N_{w}+1\right)}\right)-h_{w} \sum_{j=1}^{N_{w}}\left(r_{O j}^{2}-r_{I j}^{2}\right)\right]}{\left\{\frac{1}{2 \pi} \cdot \sum_{j=1}^{N_{w}}\left[h_{w} \cdot(1 \pm \alpha)+2 \cdot r_{I j} \cdot \ln \left(\frac{1}{\alpha}\right)\right]\right\}^{2}}
\end{aligned}
$$

Figure 6(b) presents the relationship between the normalized inductance-per-turn ${ }^{2}$ and uniformity factor when the normalized core thickness $h_{c}$ varies from 0.2 to 1 , and the number of winding windows changes from $1-4$. Compared to Figure 6(a), although adding more winding windows gives higher energy density, the inductance-perturn $^{2}$ reduces with the increased number of winding windows because the flux generated by the one winding window is not fully coupled by the other winding windows.

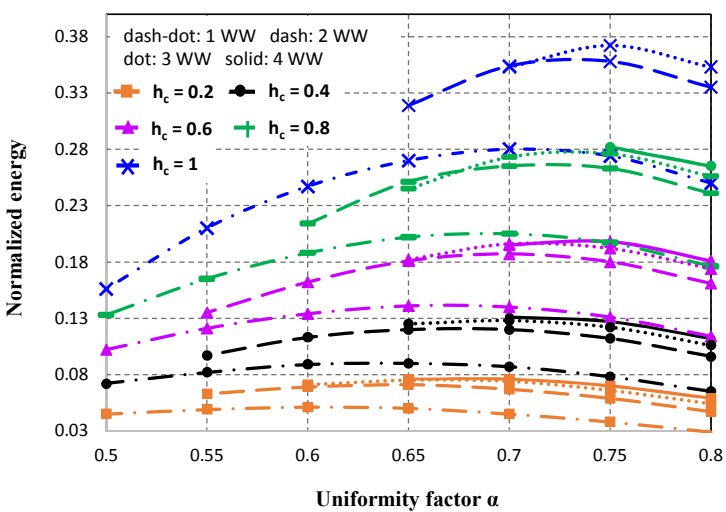

(a)

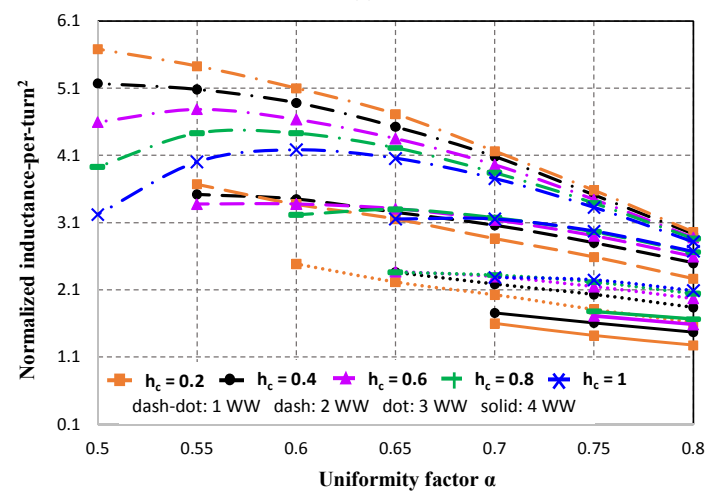

(b)

Figure 6. Impact of uniformity factor $\alpha$ on (a) normalized energy and (b) normalized inductance-per-turn ${ }^{2}$ calculated from (18) and (19) respectively using the normalized dimensions shown in Figure 3 for one to four winding windows, parametric with $h_{c}$ from 0.2 to 1 .

\section{B. Dc Winding Loss and Dc Resistance-per-Turn ${ }^{2}$}

The total dc winding loss is derived by adding up the winding loss in each winding window:

$$
P_{\text {loss }}=\frac{2 \pi \rho}{H_{w} \ln (1 / \alpha)} \cdot H_{\max }^{2} \cdot \sum_{j=1}^{N_{w}}\left[(1 \pm \alpha) H_{w}+2 R_{l j} \ln (1 / \alpha)\right]^{2}
$$

The base of dc winding loss is selected assuming $A T_{\text {base }}$ flows through a conductor of length $R_{c}$ and cross-sectional area $\pi R_{c}{ }^{2}$ as shown in (4). Thus, the normalized winding loss takes the form

$$
\begin{aligned}
p_{\text {loss }}= & P_{\text {loss }} / P_{\text {loss_base }} \\
& =\left[2 h_{w} \ln (1 / \alpha)\right]^{-1} \cdot \sum_{j=1}^{N_{w}}\left[(1 \pm \alpha) h_{w}+2 r_{I j} \ln (1 / \alpha)\right]^{2}
\end{aligned}
$$

The normalized winding loss is independent of winding resistivity and Ampere-turns. It only relates to the 
geometrical parameters like $h_{w}$ and $R_{I j}$ that are calculated from $h_{c}$ and uniformity factor $\alpha$ in (9) - (13). Figure 7(a) shows the normalized winding loss under uniformity factor from 0.5 to $0.8, h_{c}$ from 0.2 to 1 , and $1-4$ winding windows. As the uniformity factor increases, the width of a winding window becomes smaller as shown in Figure 3, and the winding loss increases. However, the plate thickness increases when $\alpha$ becomes smaller, so the winding thickness $h_{w}$ decreases with the decrease of uniformity factor. The winding loss also increases when the uniformity factor is relatively small.

The normalized resistance-per-turn ${ }^{2}$ is related to the winding loss and Ampere-turns by

$$
\begin{aligned}
& R_{N_{-} \text {norm }}=R_{N} / R_{N_{-} \text {base }}=p_{\text {loss }} / A T_{\text {norm }}^{2} \\
& =\frac{2 \pi^{2} \cdot \sum_{j=1}^{N_{w}}\left[(1 \pm \alpha) h_{w}+2 r_{l j} \ln (1 / \alpha)\right]^{2}}{h_{w} \ln (1 / \alpha) \cdot\left\{\sum_{j=1}^{N_{w}}\left[h_{w} \cdot(1 \pm \alpha)+2 \cdot r_{I j} \cdot \ln \left(\frac{1}{\alpha}\right)\right]\right\}^{2}}
\end{aligned}
$$

Figure 7(b) presents the relationship between the normalized resistance-per-turn ${ }^{2}$ and uniformity factor when the normalized core thickness $h_{c}$ varies from 0.2 to 1 , and the number of winding windows changes from $1-4$. Compared with Figure 7(a), adding more winding windows results in higher winding loss but smaller resistance-per$\operatorname{turn}^{2}$ because of the square function on the increased number of turns.

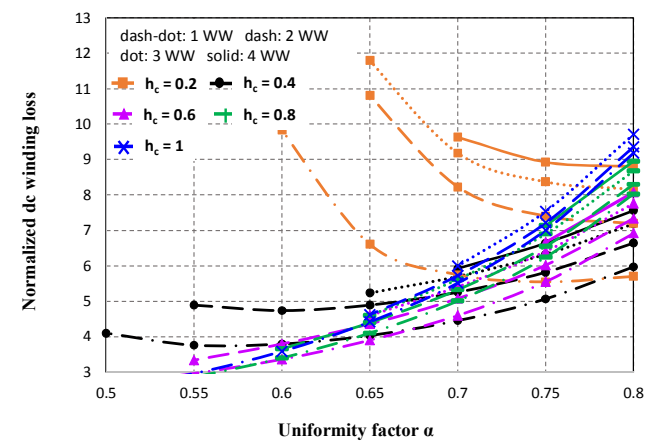

(a)

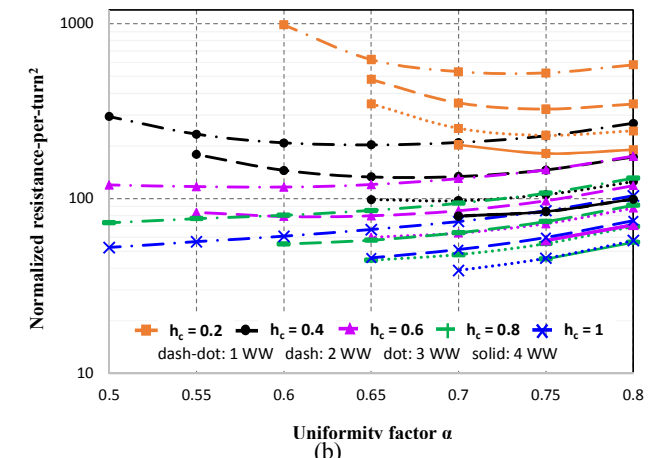

(b)

Figure 7. Impact of uniformity factor $\alpha$ on (a) normalized winding loss calculated from (21) and (b) normalized resistance-per-turn ${ }^{2}$ calculated from (22) using the normalized dimensions shown in Figure 3 for one to four winding windows, parametric with $h_{c}$ from 0.2 to 1 .

\section{Dc Quality Factor}

The dc quality factor is defined as follows to evaluate an inductor's de performance:

$$
Q_{d c}=\frac{\omega_{0} L}{R_{d c}} \quad \omega_{0}=1 \mathrm{rad} / \mathrm{s}
$$

The inductance is normalized to the inductance-per-turn ${ }^{2}$ and the dc resistance is normalized to resistance-per-turn ${ }^{2}$, so the normalized dc quality factor takes the form

$$
q_{d c}=E_{\text {norm }} / p_{\text {loss }}=A_{L_{-} \text {norm }} / R_{N_{-} \text {norm }}
$$

Figure 8 shows the plot of dc quality factor under different $h_{c}, \alpha$, and winding windows. More winding windows does not necessarily gives higher dc quality factor, and a peak value exists at the optimal uniformity factor. The peak of $q_{d c}$ is caused by the peak in the energy plot (Figure $6(\mathrm{a})$ ) and the valley in the winding loss plot (Figure 7(a)). Neither too small nor too large value of $\alpha$ is desirable for an optimal design. The optimal $\alpha$ moves to higher values when the $h_{c}$ becomes smaller, implying uniform flux is more beneficial for planar inductors with low profile. As an example, the dc quality factor of a series of commercial inductors [10] with different current rating but same package are calculated, and compared with corresponding constant-flux inductors with the same package size and inductance. As shown in Figure 9, a factor of two improvement on the $Q_{d c}$ is obtained for the whole series of inductors thanks to the constant-flux distribution. The discrepancy between the ideal curve and real data points of constant-flux inductor is caused by the approximation in the number of turns as discussed in [9].

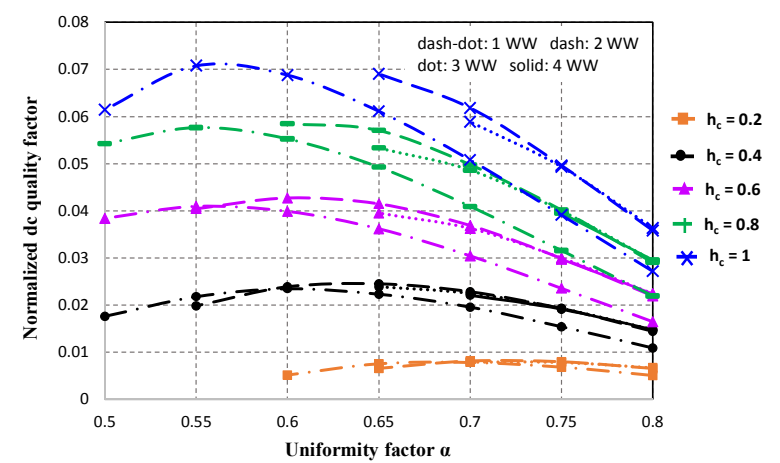

Figure 8. Impact of uniformity factor $\alpha$ on normalized dc quality factor calculated from (24) using the normalized dimensions shown in Figure 3 for one to four winding windows, parametric with $h_{c}$ from 0.2 to 1 .

\section{NORMALIZED DESIGN PROCEDURE WITH LINEAR MAGNETIC CORE}

TABLE II shows an example of normalized design procedure. In order to, the electrical inputs are given to meet the same specification of a commercial inductor [11], including target inductance $L_{r e q}$, rated current $I_{r e q}$, and target dc resistance $R_{\text {req. }}$ Practically, $R_{\text {req }}$ is calculated from the loss budget and $I_{\text {req }}$. The normalized $h_{c}$ can be obtained from the mechanical inputs include the footprint radius $R_{c}$ and inductor 


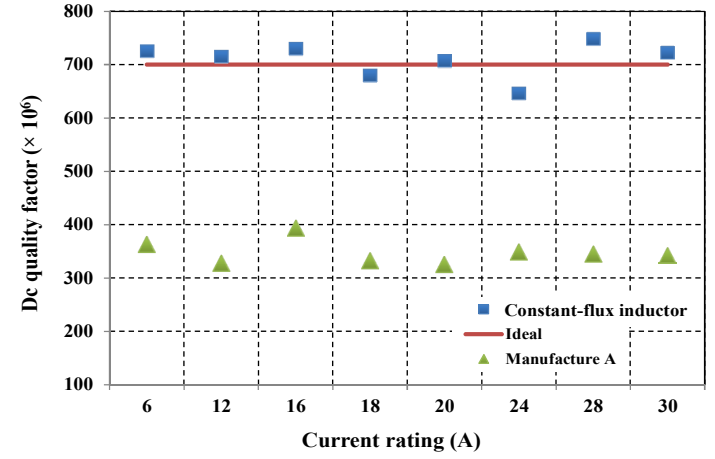

Figure 9. Comparison of dc quality factor between a series of commercial inductors [10] with a package size of $10 \times 10 \times 4 \mathrm{~mm}^{3}$ and constant-flux inductors with same inductance and package size for differnent current ratings.

height $H_{c}$ using (8). The magnetic inputs determined by the material property include maximum magnetic flux density $B_{\max }$ and permeability $\mu$. From the required inductance, resistance, and rated current (exampled shown in TABLE II), the total energy required and maximum dc winding loss allowed can be calculated. After normalizing the energy and winding loss requirement to $E_{\text {norm }}$ and $p_{\text {loss }}$, the number of winding windows can be selected based on Figure 6(a) and Figure 7(a). For Figure 6(a), the number of winding window should be large enough to ensure the energy is larger than $E_{\text {norm }}$ but should not exceed a certain value so that the winding loss is smaller than $p_{\text {loss }}$ from Figure 7(a). After the number of winding window is determined, Figure 8 helps selecting the uniformity factor that maximize the dc quality factor. When the $N_{w}$ and $\alpha$ are determined, the Ampere-turns in Figure 4 divided by the current rating yields the number of turns of winding $(N)$. The Ampere-turns $A T_{j}$ and number of turns $n_{j}$ in each winding window can be found by calculating the difference between two adjacent curves in Figure 4. Finally, the geometrical parameters can be read from Figure 3. TABLE II lists the normalized design parameters in the order of design procedure for an example targeting at $2 \mu \mathrm{H}, 11 \mathrm{~A}$, and $8 \mathrm{~m} \Omega$. The real values of the inputs and outputs for this example are summarized in TABLE III. The designed constant-flux inductor has 2.07 $\mu \mathrm{H}$ inductance and $7.0 \mathrm{~m} \Omega$ resistance within a volume of 10 $\times 10 \times 2 \mathrm{~mm}^{3}$; note that a commercial inductor [11] would occupy two time the volume with the same inductance, dc resistance, and current rating.

\section{CONSIDERATIONS OF SATURATION AND TEMPERATURE RISE}

Two current ratings are defined for a commercial inductor based on heat and saturation. One, "Heat Rating Current" or $I_{d c}$, is the dc current that causes a temperature rise of $40^{\circ} \mathrm{C}$. The other, "Saturation Rating Current" or $I_{\text {sat }}$, is the current that causes the inductance to drop to $80 \%$ of the value measured at zero dc current because of saturation in the core. Uniform flux distribution indeed improves these ratings as demonstrated below.
TABLE II. EXAMPLE OF NORMALIZED DESIGN ProcedURE

\begin{tabular}{|c|c|c|}
\hline Parameter & Design method & Value \\
\hline$L_{\text {req }}$ & Specified & $2 \mu \mathrm{H}$ \\
\hline$I_{\text {req }}$ & Specified & $11 \mathrm{~A}$ \\
\hline$R_{\text {req }}$ & Specified & $8 \mathrm{~m} \Omega$ \\
\hline$h_{c}$ & $\begin{array}{c}\text { From }(8) \text { with } R_{c}=5 \mathrm{~mm} \text { and } H_{c}=2 \mathrm{~mm} \\
\text { specified }\end{array}$ & 0.4 \\
\hline$B_{\max }, \mu$ & Specified from magnetic material property & $0.35 \mathrm{~T}, 22 \mu_{0}$ \\
\hline$E_{\text {req }}$ & $E_{\text {req }}=1 / 2 L_{\text {req }} I_{\text {req }}^{2}$ & $1.21 \times 10^{-4} \mathrm{~J}$ \\
\hline$P_{\text {loss_max }}$ & $P_{\text {loss_max }}=I_{\text {req }}^{2} R_{\text {req }}$ & $0.968 \mathrm{~W}$ \\
\hline$E_{\text {base }}$ & From $(3)$ & $8.7 \times 10^{-4} \mathrm{~J}$ \\
\hline$P_{\text {base }}$ & From $(4)$ & $0.17 \mathrm{~W}$ \\
\hline$Q_{d c \text { base }}$ & $Q_{d c \_b a s e}=E_{\text {base }} / P_{\text {base }}$ & $5.1 \times 10^{-3}$ \\
\hline$E_{\text {norm }}$ & From $E_{\text {norm }}=E_{\text {req }} / E_{\text {base }}, E_{\text {base }}$ from (3) & 0.139 \\
\hline$p_{\text {loss }}$ & $\begin{array}{c}\text { From } p_{\text {loss }}=P_{\text {loss_max }} / P_{\text {loss_base }}, P_{\text {loss_base }} \\
\text { from }(4)\end{array}$ & 5.7 \\
\hline$N_{w}$ & $\begin{array}{c}\text { Determined from Figure } 6(\mathrm{a})\left(\mathrm{N}_{\mathrm{w}}>2\right) \text { and } \\
\text { Figure } 7(\mathrm{a})\left(\mathrm{N}_{\mathrm{w}}<4\right)\end{array}$ & 3 \\
\hline$q_{d c}$ & $q_{d c}=E_{\text {norm }} / P_{\text {loss_norm }}$ & 0.024 \\
\hline$\alpha$ & Determined from Figure 8 & 0.65 \\
\hline$N$ & Calculated from Figure 4 and (2) & 8 \\
\hline$r_{O j}$ & Read from Figure 3(a) & $0.9,0.54,0.3$ \\
\hline$r_{l j}$ & Read from Figure 3(b) & $0.58,0.34,0.2$ \\
\hline$h_{p}$ & Read from Figure 3(d) & 0.1 \\
\hline
\end{tabular}

TABLE III. DESIGN PARAMETERS OF CONSTANT-FLUX INDUCTOR WITH SAME L AND R IN HALF VOLUME OF A COMMERCIAL INDUCTOR [10]

\begin{tabular}{|c|c|c|c|c|c|c|}
\hline \multicolumn{7}{|c|}{ INPUTS } \\
\hline $\begin{array}{l}\mathrm{B}_{\max } \\
(\mathrm{T})\end{array}$ & $\begin{array}{c}\mathrm{R}_{\mathrm{c}} \\
(\mathrm{mm})\end{array}$ & $\begin{array}{c}\mathrm{H}_{\mathrm{c}} \\
(\mathrm{mm})\end{array}$ & $\begin{array}{l}\mathrm{I}_{\mathrm{r}} \\
\text { (A) }\end{array}$ & $\begin{array}{c}\mathrm{L}_{\mathrm{req}} \\
(\mu \mathrm{H})\end{array}$ & $\begin{array}{c}\mathrm{R}_{\mathrm{req}} \\
(\mathrm{m} \Omega)\end{array}$ & $\begin{array}{c}\mu \\
\left(\mu_{0}\right)\end{array}$ \\
\hline 0.35 & 5 & 2 & 11 & 2 & 8 & 22 \\
\hline \multicolumn{7}{|c|}{ OUTPUTS $(\alpha=0.65)$} \\
\hline \multicolumn{2}{|c|}{ Winding Windo } & \multicolumn{2}{|c|}{$\mathrm{R}_{\mathrm{Oj}}(\mathrm{mm})$} & $\mathrm{R}_{\mathrm{Ij}}(\mathrm{mm})$ & $\mathrm{AT}_{\mathrm{j}}(\mathrm{A})$ & $\mathrm{n}_{\mathrm{j}}$ \\
\hline \multicolumn{2}{|r|}{$\mathrm{j}=1$} & \multicolumn{2}{|c|}{4.5} & 2.9 & 52.6 & 4 \\
\hline \multicolumn{2}{|r|}{$j=2$} & \multicolumn{2}{|c|}{2.7} & 1.7 & 23.7 & 2 \\
\hline \multicolumn{2}{|r|}{$\mathrm{j}=3$} & & & 1.0 & 15.3 & 2 \\
\hline \multicolumn{4}{|c|}{ Total thickness of winding $\left(\mathrm{H}_{\mathrm{w}}\right)$} & \multicolumn{3}{|c|}{$1 \mathrm{~mm}$} \\
\hline \multicolumn{4}{|c|}{ Inductance calculated (L) } & \multicolumn{3}{|c|}{$2.07 \mu \mathrm{H}$} \\
\hline \multicolumn{4}{|c|}{ Dc resistance calculated $\left(\mathrm{R}_{\mathrm{dc}}\right)$} & \multicolumn{3}{|c|}{$7.0 \mathrm{~m} \Omega$} \\
\hline
\end{tabular}

\section{A. Improvement of Heat Rating Current}

The improvement of dc quality factor in Figure 9 resulted from the two times smaller resistance of constantflux inductor. The reduction of dc resistance leads to reduction of dc loss; therefore, the temperature rise caused by dc winding loss would be smaller. In addition, the distribution of the winding turns throughout the core volume shortens the thermal path between the heat source and the core surface, further reducing temperature rise. In order to compare the thermal performance of the constant-flux inductor with the commercial inductor shown in Figure 1(a) with the same volume and inductance, the two inductors were simulated in finite-element analysis (FEA) software with the same convection conditions (convection coefficient $\left.=50 \mathrm{~W} / \mathrm{m}^{2} / \mathrm{K}\right)$ ) and same magnetic material property (thermal conductivity $=20 \mathrm{~W} / \mathrm{m} / \mathrm{C}$ ). Since the constant-flux 
inductor has varying width of winding turns, different thermal-load density was applied to each turn while the commercial inductor with same winding width had the same thermal-load density on the winding turns. Figure 10 compares the simulation results for the CFI and commercial inductor [10]. Since the winding loss of the CFI is two times smaller than that of the commercial product, the temperature rise at the surface of CFI is also about two times smaller than the commercial product without seeing any hot spot areas, core loss not considered in the thermal simulation. Therefore, the current rating $I_{d c}$ is improved by $50 \%$ for the constant-flux inductor.

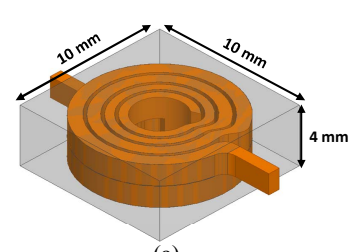

(a)

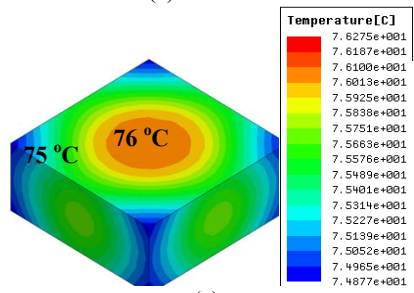

(c)

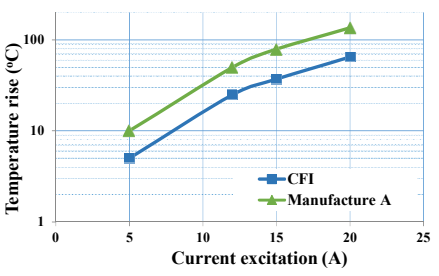

(b)

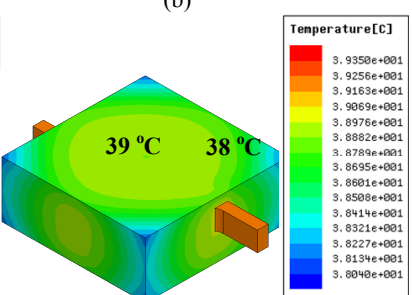

(d)
Figure 10. (a) Constant-flux inductor (CFI) for simulation; (b) temperature rise versus current excitation of the commercial inductor shown in Figure 1(a) with $2.2 \mu \mathrm{H}$ inductance, $7 \mathrm{~m} \Omega$ resistance, and a volume of $10 \times 10 \times 4 \mathrm{~mm}^{3}$, and CFI with $2.2 \mu \mathrm{H}$ inductance, $3.2 \mathrm{~m} \Omega$ resistance within the same footprint and thickness; thermal maps of (c) commercial inductor and (d) CFI.

\section{B. Improvement of Saturation Rating Current}

In order to explore the impact of constant-flux distribution on the saturation current rating $I_{\text {sat }}$, the commercial inductor and the constant-flux inductor were simulated with same material property, and the inductances under different current excitations were recorded. The nonlinear B-H curve of Micrometals No. -8 material [12] with an initial permeability of $35 \mu_{0}$ was used to simulate the two structures. As shown in Figure 11, the saturation current of commercial product is $13 \mathrm{~A}$ while the constant-flux inductor has a saturation current of $16 \mathrm{~A}$, which is $23 \%$ higher than the commercial product.

The reason of the increased saturation current can be examined and explained by the magnetic field distribution. Since the commercial inductor has non-uniform flux distribution, as the current increases, some area of the core saturates first and brings down the permeability as a result [13]. When the current excitation is $20 \mathrm{~A}$, a large area of the core has very high flux density (see Figure 1(b)), the permeability drops to a lower value (see Figure 12(a)), and the inductance is reduced significantly. However, for the constant-flux inductor, the flux density still remains uniform and lower than the saturation flux density, and the permeability still remains a relatively high value as shown in Figure 12(b). Therefore, the magnetic field with uniform flux distribution always saturates slower than the one with non-uniform flux distribution.

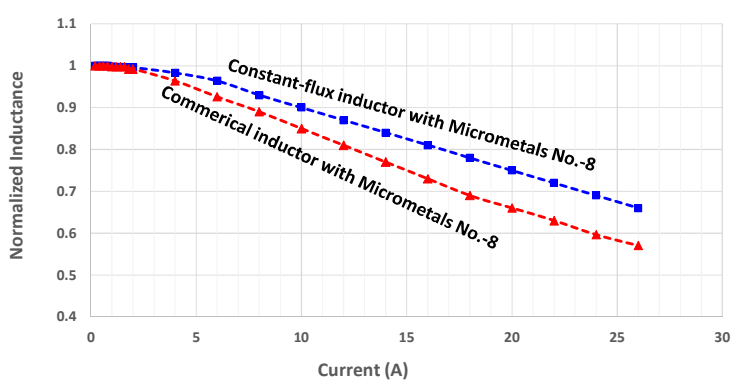

Figure 11. Comparisons of normalized inductance under different current excitation with Micrometals No. -8 material for commercial inductor and constant-flux inductor.

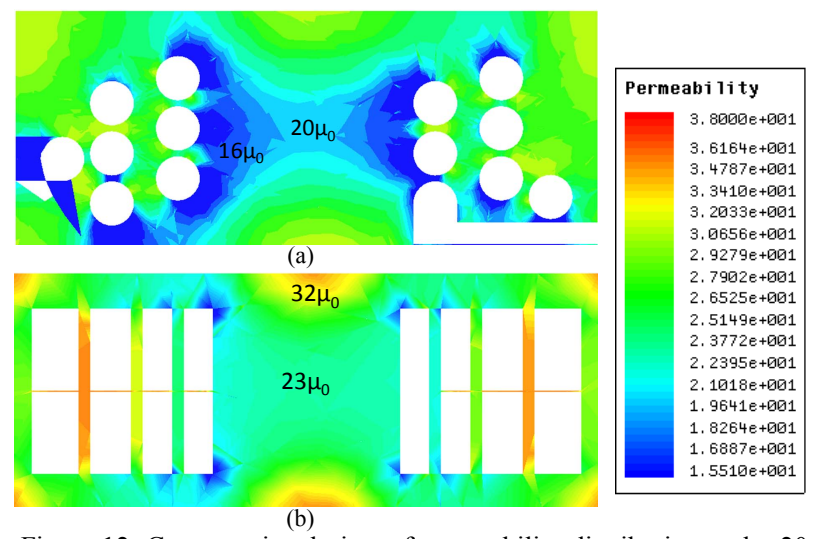

Figure 12. Cross-sectional view of permeability distribution under 20 A current excitation for commercial inductor (a) and constant-flux inductor (b) with B-H curve of Micrometals -8 material.

\section{EXPERIMENTAL VERIFICATION}

The concept was validated by an inductor prototype made with powder-iron material and copper sheet [9]. The measured inductance and dc resistance of the fabricated constant-flux inductor prototype herein are $0.88 \mu \mathrm{H}$ and 8 $\mathrm{m} \Omega$, respectively.

In order to measure the inductance versus dc current, the inductor prototype was put into a buck converter as shown in Figure 13(a) with $3.4 \mathrm{~V}$ input voltage and $2.5 \mathrm{~V}$ output voltage. The input voltage was generated by TDK-Lambda GEN60-25 power supply, and the output voltage was measured by Tektronix TDP 1000 differential probe. The switching frequency was $1.5 \mathrm{MHz}$ so that the current ripple was small enough to measure the incremental inductance under different dc current. The dc current through the inductor was controlled by changing the electronic load (TDI RBL488 100-120-800) under constant-current mode and the inductor current was measured by Tektronix TCP0030 current probe. The inductance was therefore calculated from the measured voltage and current on the inductor. Figure 13(b) shows the experimental setup for the 
inductance measurement. When the dc current changes from $1 \mathrm{~A}$ to $14 \mathrm{~A}$, the measured inductance matches well with the simulated result with a maximum error of $3.8 \%$, as shown in Figure 13(c).

\section{CONCLUSION}

In this paper, the optimal design of a constant-flux inductor that maximize the inductor's performance has been discussed. The design procedure of the constant-flux inductor is normalized so that the result is more general. In addition, the normalization decouples geometrical dimensions and magnetic material properties in design and evaluation. The heat rating current is improved by $50 \%$ because the dc quality factor of the constant-flux inductor is two times larger compared to a commercial product with the same volume and inductance. The saturation rating current is also improved by $20 \%$ resulted from the uniform flux distribution inside the core. The simulation result of saturation current is verified by the measurement results on the fabricated inductor prototype under different dc currents.

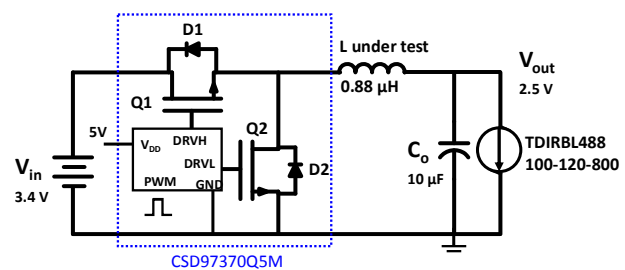

(a)

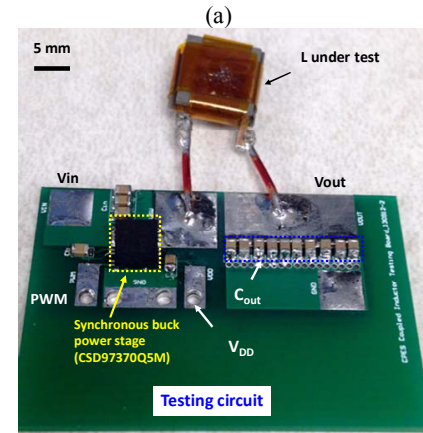

(b)

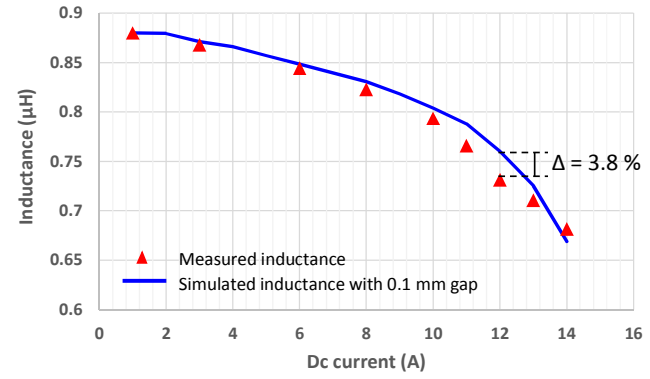

Figure 13. (a) Testing circuit of synchronous buck converter for inductance measurement under dc bias; (b) Experimental setup for inductance measurement versus dc current in a buck converter; (c) comparisons between measurement and simulation result of inductance under different current excitations for the constant-flux inductor prototype.

\section{REFERENCES}

[1] K. D. T. Ngo, R. P. Alley, A. J. Yerman, R. J. Charles, and M. H. Kuo, "Design issues for the transformer in a low-voltage power supply with high efficiency and high power density," IEEE Trans. Power Electron., vol. 7, no.3, pp. 592 - 600, Jul. 1992.

[2] K. D. T. Ngo, E. Alpizar, and J. K. Watson, "Modeling of losses in a sandwiched-winding matrix transformer," IEEE Trans. Power Electron., vol. 10, no. 4, pp. 427 - 434, Jul. 1995.

[3] E. Osegueda, K. D. T. Ngo, W. M. Polivka, and M. M. Walters, "Perforated-plate magnetics. Part I: Mode-1 inductor/transformer," IEEE Trans. Aerosp. Electron. Syst., vol. 31, no. 3, pp. 968 - 976, Jul. 1995

[4] E. Herbet, "Flat Matrix Transformers", US Patent 4, 665, 357, 12 May 1987.

[5] L. Wang, Y. Pei, Y. Xu, Z. Wang, and Y. Liu, "A horizontal-winding multi-permeability distributed air-gap inductor," in Proc. IEEE Appl. Power Electron. Conf. Expo., 2012, pp. 994 - 1001.

[6] Q. Li and F. C. Lee, "High Inductance Density Low-Profile Inductor Structure for Integrated Point-of-Load Converter," in Proc. IEEE Appl. Power Electron. Conf. Expo., 2009, pp. 1011 - 1017.

[7] J. K. Seshadri, D. Xu, and K. D. T. Ngo, "Inductor structure with improved space utilization," Electronics Letters, vol. 48, no. 3, pp. 164 - 165, Feb. 2012

[8] H. Cui and K. D. T. Ngo, "Constant-flux inductor with enclosed winding for high-density energy storage," Electronics Letters, vol. 49, no. 13, pp. 841 - 843, June. 2013.

[9] H. Cui, K. D. T. Ngo, J. Moss, M. H. Lim, and E. Rey, "Inductor geometry with improved energy density," IEEE Trans. Power Electron., vol. 29, no. 10, pp. 5446 - 5453, Oct. 2014.

[10] Ultra high current inductor MMD - 12DZ - 2R2M - V1 datasheet. (2007) [online]. Available: http://www.maglayersusa.com/

[11] High current shielded SMD power inductor SCRH104R datasheet. [online]. Available: http://www.signaltransformer.com/sites/all/pdf/ smd/P150_SCRH104R.pdf

[12] Nonlinear B-H curve of power magnetic material No. -8 , [online] Available: http://www.micrometals.com/materials_index.html

[13] R. W. Erickson and D. Maksimovic, Fundamentals of Power Electronics, $2^{\text {nd }}$ ed. Boston, MA: Kluwer, 2000, pp. 506 - 507. 